\title{
Changes in plasma von Willebrand factor and von Willebrand factor cleaving protease in thrombotic thrombocytopenic purpura: A case report
}

\author{
YU GAO, LIPING WANG and GUANGXIAN NAN
}

Second Department of Neurology, China-Japan Union Hospital of Jilin University, Changchun, Jilin 130033, P.R. China

Received February 16, 2016; Accepted July 20, 2017

DOI: $10.3892 /$ etm.2017.5361

\begin{abstract}
The present case report described a patient with thrombotic thrombocytopenic purpura (TTP), who presented to the China-Japan Union Hospital of Jilin University (Changchun, China), and the changes in plasma von Willebrand factor (vWF) and vWF cleaving protease (vWF-cp) observed during treatment. Computed tomography and diffusion-weighted magnetic resonance (MR) imaging of the brain, cerebral MR angiography and other experimental tests were performed and the patient was subsequently diagnosed with TTP. The patient underwent treatment with plasma exchange, glucocorticoids and supportive care. Hematologic and biochemical parameters began to gradually improve over the 12 days after admission; platelet count, serum creatinine and serum lactate dehydrogenase concentrations returned to their normal ranges, plasma vWF concentration decreased to normal levels over the 30 days after admission, and vWF-cp activity increased compared with the levels detected in healthy volunteers. Monthly rituximab treatment was administered 4 times following patient discharge to prevent relapse, and no recurrence was detected at the 20-month follow-up. Plasma exchange therapy is effective in patients with TTP. After low-dose rituximab treatment, recurrent TTP has not been detected found till now. In the present case, $\mathrm{vWF}$ concentration and vWF-cp activity were measured at 8, 10, 23 and 32 days after admission; compared with the control group, patient's vWF concentration gradually decreased and vWF-cp activity slowly increased, suggesting that the patient had a favorable prognosis and a low risk of recurrence.
\end{abstract}

\section{Introduction}

Thrombotic thrombocytopenic purpura (TTP) is a rare thrombotic microangiopathy (TMA) that may cause microangiopathic

Correspondence to: Dr Guangxian Nan, Second Department of Neurology, China-Japan Union Hospital of Jilin University, 126 Xiantai Street, Changchun, Jilin 130033, P.R. China E-mail:ngx0415@126.com

Key words: thrombotic thrombocytopenic purpura, plasma exchange, plasma von Willebrand factor, von Willebrand factor cleaving protease hemolytic anemia, thrombocytopenia, fever, central nervous system (CNS) symptoms and renal injury, which have been described as the TTP pentad (1). TTP has a high incidence in adults, is more common in females than males, and occurs after infection, autoimmune disease, pregnancy, medication, hematopoietic stem cell transplantation or drug allergy. The incidence of TTP in countries outside China is 3.7/1,000,000, and there is no definite statistical data in China (2). With in-depth understanding of this disease, the diagnosis rate has increased. There is an increased number of patients with TTP occurring following medication or other diseases, with the incidence of $2 / 1,000,000$ to $8 / 1,000,000$ (3). Prior to the introduction of plasma exchange (PE) treatment, patients with TTP typically had a poor prognosis; however, unlike other types of TMA, TTP may now be treated effectively by PE (4). The mortality rate of TTP has dropped from 95 to $20 \%$ since the introduction of PE treatment, but early diagnosis is critical to ensure the most optimal possible outcome for patients (4). The occurrence of TTP is associated with vascular endothelial cell injury, plasma von Willebrand factor (vWF) and vWF cleaving protease (vWF-cp). Infection, autoimmune diseases and/or medication cause vascular endothelial cell damage, release of a large number of vWF, lack of vWF-cp or inhibition of vWF-cp activity, leading to microaggregation of platelets and vWF-fibrinogen, vessel occlusion, and rapid reduction of platelets, and finally resulting in occurrence of TTP (5). The present case report described a patient with TTP, who presented to the China-Japan Union Hospital of Jilin University (Changchun, China), and the changes in vWF and vWF-cp observed during treatment.

\section{Case report}

The present study was approved by the Ethics Committee of China-Japan Union Hospital of Jilin University and informed, written consent was received from the patient in the present study. A 47-year-old man was admitted in February 2014 to the China-Japan Union Hospital of Jilin University complaining of a 19-day history of fever, and a 2-h history of right limb dysfunction and loss of speech. The patient's peak body temperature was $37.7^{\circ} \mathrm{C}$, and the patient had reported a small amount of gingival bleeding. The sudden onset of neurologic symptoms included difficulty standing, walking and holding items due to right-sided weakness, slow response to 
commands and inability to speak. His right limb symptoms had improved by the time of hospital admission but his other symptoms had not. The patient had a 2-year history of arterial hypertension, with the highest recorded blood pressure (BP) of $160 / 90 \mathrm{mmHg}$; however, he had not received treatment with antihypertensive agents.

Clinical examination on admission revealed a BP of $110 / 80 \mathrm{mmHg}$ (normal range, $80-120 / 60-80 \mathrm{mmHg}$ ) and dysarthria; cognitive function, including problem solving and memory, was impaired, MMSE 17 points (university education level, $\leq 23$ points indicative of cognitive dysfunction) (6). Computed tomography and diffusion-weighted magnetic resonance (MR) imaging of the brain, and cerebral MR angiography were performed, but no abnormalities were identified (Fig. 1). Laboratory investigations indicated a white blood cell (WBC) count of $12.8 \times 10^{9}$ cells $/ 1$ (reference range, $4-10 \times 10^{9}$ cells $/ 1$ ), a red blood cell (RBC) count of $2.5 \times 10^{12}$ cells/l (reference range, $3.5-5.5 \times 10^{12}$ cells $\left./ \mathrm{l}\right)$, a hemoglobin $(\mathrm{Hb})$ concentration of 75.0 g/l (reference range, 110-160 g/l) and platelet (PLT) count of $11 \times 10^{9}$ cells $/ 1$ (reference range, $100-300 \times 10^{9}$ cells $/ 1$ ). Urinalysis revealed a WBC count of 107.1 cells $/ \mu 1$ (reference range, $0-25$ cells $/ \mu \mathrm{l}$ ) and a RBC of 84.2 cells $/ \mu 1$ (reference range, $0-25$ cells $/ \mu \mathrm{l})$. Dipstick urinalysis detected protein $2+(\mathrm{Neg})$, occult blood 3+ $(\mathrm{Neg})$, urobilinogen $1+(\mathrm{Neg})$ and urobilin $1+(\mathrm{Neg})$. Liver function tests revealed serum concentrations of alanine transaminase of $323 \mathrm{IU} / \mathrm{l}$ (reference range, 5-40 IU/1), aspartate aminotransferase of $114 \mathrm{IU} / 1$ (reference range, 8-40 IU/l), $\gamma$-glutamyl transferase of $72 \mathrm{IU} / 1$ (reference range, 8-57 IU/1) and glutamate dehydrogenase of $35 \mathrm{U} / 1$ (reference range, 0-10 U/l), a total bilirubin concentration of $46.0 \mu \mathrm{mol} / 1$ (reference range, $5-21 \mu \mathrm{mol} / \mathrm{l}$ ), a direct bilirubin concentration of $11.5 \mu \mathrm{mol} / 1$ (reference range, $0-3 \mu \mathrm{mol} / \mathrm{l}$ ) and an indirect bilirubin concentration of $34.5 \mu \mathrm{mol} / 1$ (reference range, 2-21 $\mu \mathrm{mol} / \mathrm{l}$ ). Renal function tests revealed serum concentrations of urea of $12.4 \mu \mathrm{mol} / \mathrm{l}$ (reference range, 3.2-7.1 $\mu \mathrm{mol} / \mathrm{l}$ ) and creatinine (SCr) of $126.7 \mu \mathrm{mol} / 1$ (reference range, 58-110 $\mu \mathrm{mol} / \mathrm{l}$ ). A peripheral blood film (5) indicated mature erythrocytes of a variety of sizes with RBC debris and scattered microspherocytes. The reticulocyte count was $0.391 \times 10^{12}$ cells $/ 1$ (5), which accounted for $22 \%$ of the total RBCs. Bone marrow examination suggested aplastic anemia and thrombocytopenia (5). Antinuclear antibodies were not detected (5). Based on these findings, a diagnosis of TTP and grade 2 hypertension (1999 WHO/ISH Guidelines for the Management of Hypertension) (7) (very high-risk group) were determined.

A total of 4 days following admission, the patient underwent PE for the first time: cell harvesting was performed using Cobe Spectra cell separator (Terumo BCT, Inc., Lakewood, CO, USA). Frozen plasma fluid (2,000-3,000 ml) was replaced once a day for 13 days. Plasma was replaced at $15-20 \mathrm{ml} / \mathrm{min}$ and blood flow velocity was $120-150 \mathrm{ml} / \mathrm{min}$. After low-dose low molecular weight heparin anticoagulation, $30 \mathrm{~min}$ after plasma replacement, $10 \%$ calcium gluconate $(10 \mathrm{ml} / \mathrm{h})$ was intravenously administered. Glucocorticoids (Pfizer, Inc., New York, NY, USA) were administered (1.0 g once daily for 3 days, $0.5 \mathrm{~g}$ once daily for 3 days, $0.24 \mathrm{~g}$ once daily for 3 days, $0.12 \mathrm{~g}$ once daily for 3 days, $0.08 \mathrm{~g}$ once daily for 3 days and $0.04 \mathrm{~g}$ once daily for 3 days, all via intravenous drip) and supportive care was given (roton pump inhibitor, potassium, fat milk and compound amino acids). As shown in Fig. 2, patients were administered PE therapy starting from 4 days after admission, once a day, for a total of 13 days. A total of 2 days after $\mathrm{PE}$ administration, the patient sustained a cardiac arrest and required closed-chest cardiac massage and cardiopulmonary resuscitation, and an autonomous cardiac rhythm and respiration were restored. A total of 14 days following admission, a regime of $100 \mathrm{mg}$ rituximab (Roche Diagnostics, Basel, Switzerland) infused intravenously once a week for 4 weeks was initiated. By the time of his discharge 6 weeks after admission, the patient had undergone 13 sessions of PE treatment. The patient was asymptomatic and exhibited no abnormal CNS signs. The complete blood count before the patient was discharged indicated a WBC count of $5.3 \times 10^{9}$ cells/l, a RBC count of $3.3 \times 10^{12}$ cells $/ 1$, a Hb concentration of $115.0 \mathrm{~g} / \mathrm{l}$ and a PLT count of $348 \times 10^{9}$ cells/l. Urinalysis and liver function tests were normal. Monthly rituximab was administered for 4 times after discharge to prevent relapse, and no recurrence was detected at the 20-month follow-up after discharge.

A 4-ml specimen of the morning fasting venous blood was obtained from the patient at different phases of disease (at 8,10,23 and 32 days after admission) to allow comparison with two healthy volunteers. These two healthy volunteers (a 33-year-old male and a 35-year-old male) were persons who received physical examination in January 2016 in the outpatient clinic of China-Japan Union Hospital of Jilin University. The volunteers signed informed consent regarding participation in this study. Blood was taken in EDTA tubes and centrifuged at $1,500 \mathrm{x} \mathrm{g}, 4^{\circ} \mathrm{C}$ for $20 \mathrm{~min}$. Supernatant plasma was collected and stored at $-20^{\circ} \mathrm{C}$ prior to measurement of $\mathrm{vWF}$ and vWF-cp concentrations. Measurements were performed using a double antibody sandwich ELISA kit (CSB-E08437h; Cusabio Biotech Co., Ltd., Wuhan, China) according to the manufacturer's protocols.

Following PE, hematologic and biochemical parameters (analyzed using an automatic biochemical analyzer) began to gradually improve over the 32 days after admission; PLT, $\mathrm{SCr}$ and serum lactate dehydrogenase concentration returned to their normal ranges. Furthermore, plasma vWF concentrations decreased and vWF-cp activity increased (Table I). On day 8 after admission, the patient's vWF concentration was increased compared with the normal control, then gradually decreased, and was close to normal level at discharge. On day 8 after admission, the patient's vWF-cp activity was markedly increased compared with the normal control, maintained at a very high level, and it did not recover to the normal level at discharge.

Once PLT had returned to the normal range $\left(193 \times 10^{9} \mathrm{cells} / \mathrm{l}\right)$ at 32 days after admission, the patient's plasma vWF concentration was broadly comparable with that of healthy volunteers. Additionally, plasma vWF-cp activity had markedly increased compared with that of healthy volunteers controls (Table I). Plasma vWF concentration decreased to normal levels over the 32 days after admission, and vWF-cp activity remained very high and did not recover to normal level at discharge (Table I).

\section{Discussion}

TTP is rare, rapidly progressive and it has very high mortality rate if plasma replacement therapy is not given (8). TTP has 
Table I. Comparison of the patient's hematologic profile with healthy controls.

Patient results according to hospital stay, days

Variable

8

23

32

Control

Control

Complete blood count

Platelets, $\mathrm{x} 10^{9}$ cells $/ 1$

White blood cells, $\mathrm{x} 10^{9}$ cells $/ 1$

Hemoglobin concentration, $g / 1$

Number of PE sessions

Number of rituximab treatments

Serum creatinine concentration, $\mu \mathrm{m} / \mathrm{ml}$

Lactate dehydrogenase, U/1

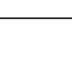

vWF, ng/ml

$\begin{array}{cccc}41 & 51 & 62 & 193 \\ 25 & 15 & 8 & 4 \\ 99 & 89 & 86 & 108 \\ 6 & 10 & 12 & 12 \\ 0 & 0 & 2 & 4 \\ 153.5 & 134.7 & 89 & 85 \\ 1,770 & 1,131 & 343 & 265 \\ 12.80 & 16.59 & 13.75 & 8.59 \\ 36.39 & 36.30 & 58.89 & 50.89\end{array}$

193

vWF-cleaving protease, $\mathrm{U} / \mathrm{ml}$

36.39

36.30

vWF, von Willebrand factor.
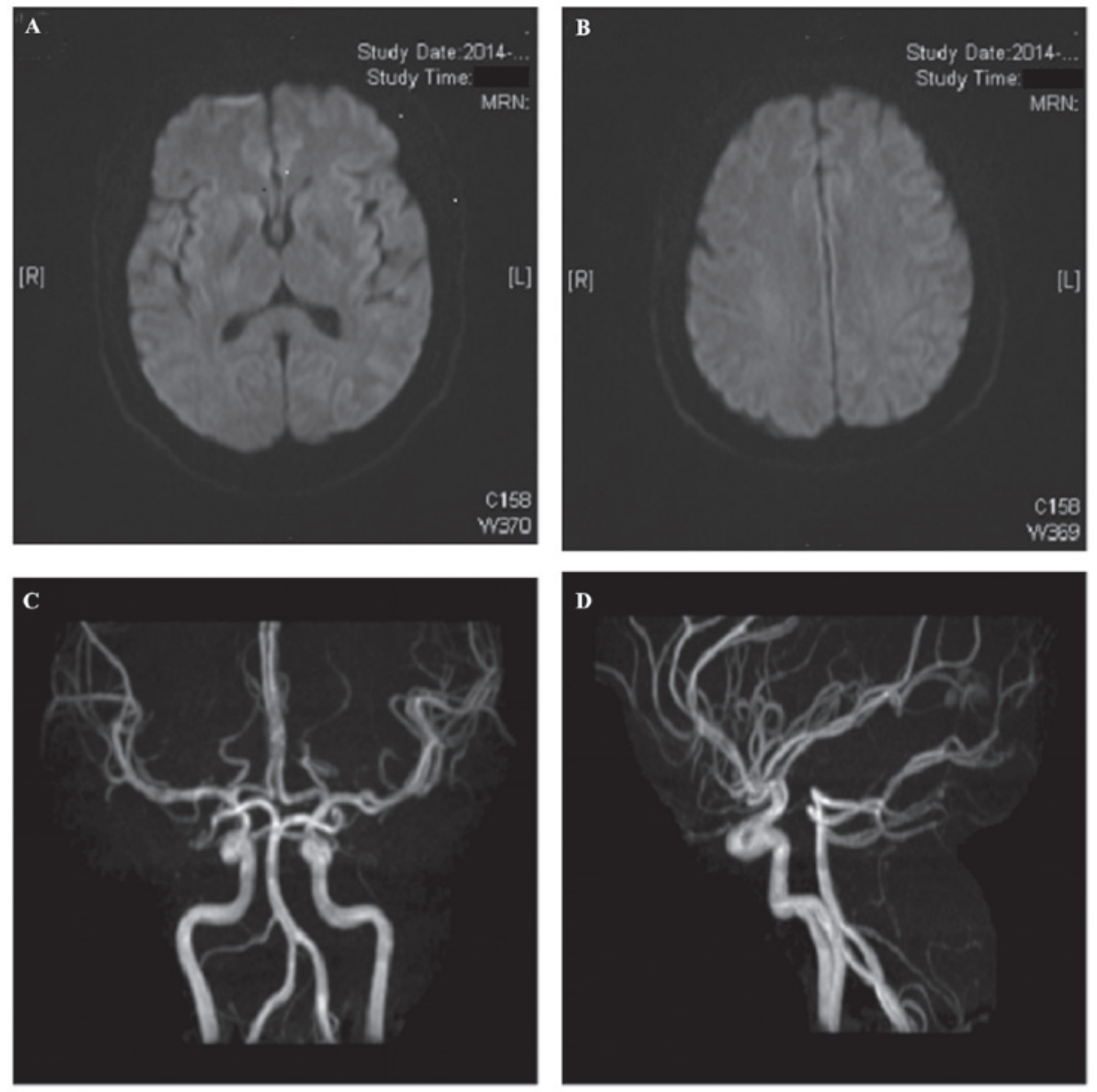

Figure 1. DWI of the brain and MRA. (A and B) 3 days after admission, DWI of the brain indicated no signal abnormalities. (C and D) 3 days after admission, MRA of the brain indicated no abnormalities. L, left; R, right; DWI, diffusion-weighted magnetic resonance imaging; MRA, magnetic resonance angiography.

been indicated to cause thrombocytopenia and thrombogenesis in the small arteries and capillaries (9). The mortality rate of TTP is $90 \%$ without PE, however, the introduction of PE has improved the mortality rate (4). A study by Bukowski et al (10) first reported the successful treatment of TTP with PE in 1976, and PE has now become the first choice treatment for TTP. Timely, extensive PE has been indicated to reduce the mortality rate to $<10 \%$, resulting in $>90 \%$ short-term effectiveness (4). PE should be sustained for 2-3 days after the restoration of PLT to $150 \times 10^{9}$ cells/1, after which it may be stopped (11). 


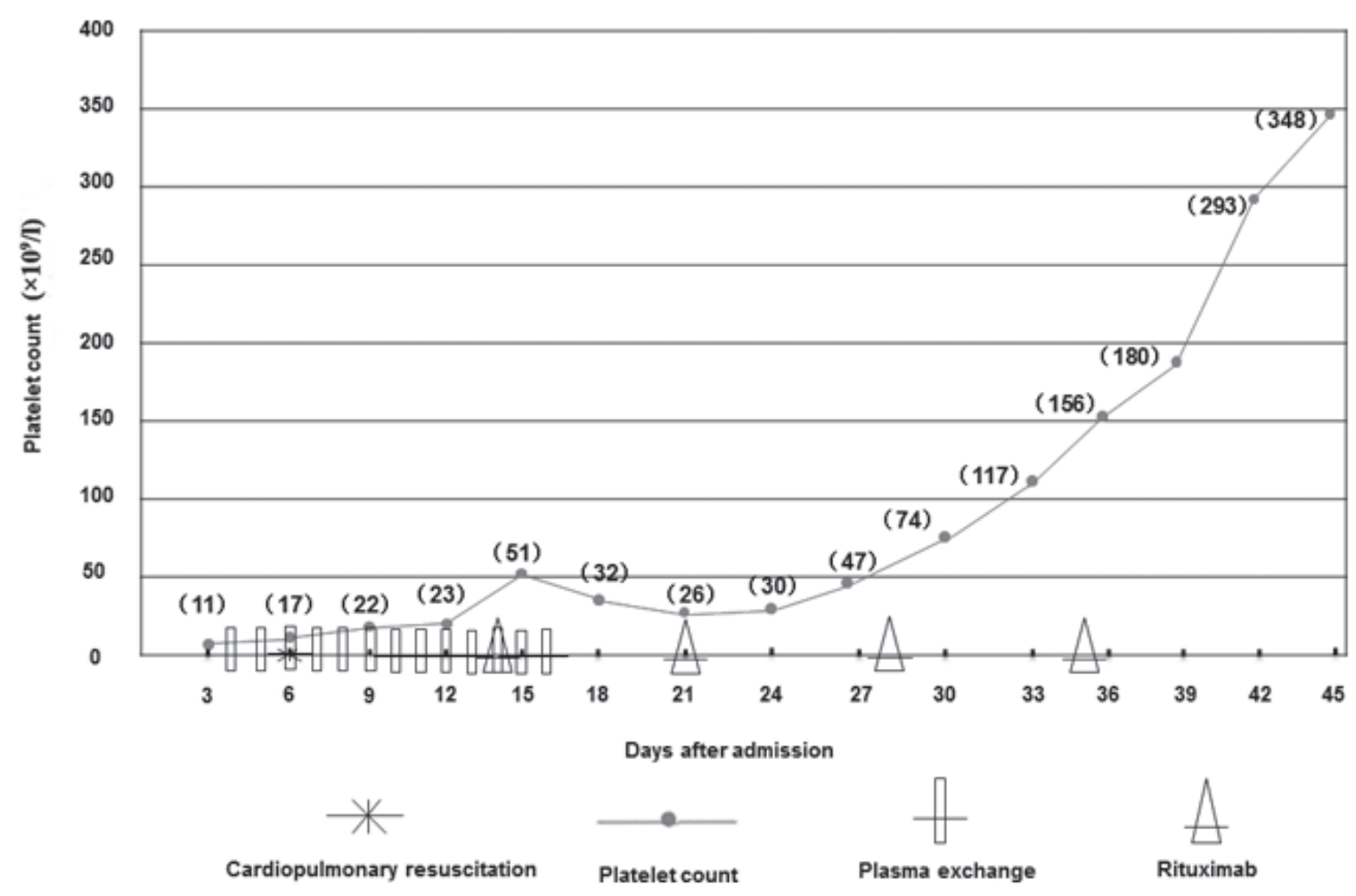

Figure 2. Clinical course of thrombotic thrombocytopenic purpura and treatments administered.

However, a longer course of treatment may be required for patients at risk of long-term relapse who respond poorly to PE (12). Although a standard dose (375 mg rituximab/m² body surface area administered once a week for 4 consecutive weeks) of rituximab may also be effective for these patients, the high cost of this treatment limits its use (13). Rituximab is a biosynthesized mouse/human chimeric monoclonal antibody that specifically binds cluster of differentiation 20 antigens on the B-lymphocyte surface, preferentially clearing those abnormally reacting with autoantigens by inducing complement-dependent cytotoxicity and antibody-dependent cell-mediated cytotoxic reactions (14). Rituximab targets and selectively removes abnormal B-lymphocytes, and has therefore been widely used for the treatment of autoimmune diseases in which B-lymphocytes are dysfunctional (14). For TTP treatment, rituximab removes the lymphocytes responsible for producing vWF (14). It has been reported that $375 \mathrm{mg}$ rituximab per square meter body surface area administered once a week for 4 consecutive weeks is a highly effective treatment for idiopathic TTP, particularly for patients with low vWF-cp activity and vWF-cp-positive antibodies, and has been indicated to result in a remission rate of $\sim 95 \%$ and a recurrence rate of $\sim 10 \%$ (14). In patients that experience TTP recurrence, further use of rituximab may improve the patient outcome (15). Given that the cost of rituximab is high, and when used to treat TTP it is administered outside its licensed indications (rituximab is typically used to treat B-lymphocyte-mediated diseases, including non-Hodgkin's lymphoma, chronic lymphocytic leukemia, rheumatoid arthritis, granulomatosis with polyangiitis and microscopic polyangiitis), it has been suggested that low-dose rituximab is a satisfactory treatment for TTP (16). In the present case study, continued administration of low-dose rituximab after $\mathrm{PE}$ was provided to the patient and no relapse was indicated at the 20-month follow-up after discharge.

A study by Moake et al (17) first detected vWF in the serum of patients with TTP in 1982, laying the foundations for the study of TTP pathogenesis. In 1996, a study by Furlan et al (18) isolated a serum metalloproteinase that cleaves vWF, and while the cause of TTP is not known, it was found that patients with TTP lack this metalloproteinase (18). In 2001, a study by Gerritsen et al (19) purified the metalloproteinase and demonstrated that it belonged to an ADAMTS gene family, the genes for vWF-cp (ADAMTS13) have been mapped to chromosome $9 \mathrm{q} 34$ (19). Previous findings have recognized that deletion of ADAMTS13 may be acquired or hereditary, but both result in sustained vWF-dependent aggregation of platelets, microthrombus formation and TTP (20). These findings suggest that TTP may be caused by relative or absolute deficiencies in the activity of plasma vWF-cp. For most patients with hereditary TTP, the vWF-cp activity is always $<5 \%$, and may be $0 \%$ in those with idiopathic TTP (21). A decrease in activity to $<5 \%$ may indicate the formation of plasma anti-vWF-cp antibodies (21). Previous results suggest that prognosis appears favorable if vWF-cp activity rises to $\sim 50 \%$ and the antibody titer falls after PE (18). In addition, although not all patients with TTP present with severe depletion of vWF-cp activity, it may still help guide treatment (22). Among TTP survivors who have a vWF-cp activity $<10 \%$, nearly half relapse, most commonly in the second year after the acute event (22). Conversely, those with normal vWF-cp activity after treatment are unlikely to suffer from recurrence (22). This suggests that any factor causing vWF-cp depletion at any time may increase the risk of recurrence for patients who have recovered from 
TTP. Some results have concluded that vWF-cp activity $>10 \%$ of the normal range identifies patients at high risk of recurrence (23). In the present case, $\mathrm{vWF}$ concentration and $\mathrm{vWF}-\mathrm{cp}$ activity was measured at 8, 10, 23 and 32 days after admission; when compared with the controls, results suggested that the patient's vWF concentration gradually decreased and vWF-cp activity slowly increased to $51 \%$, which was suggestive of a favorable prognosis and a lower risk of recurrence.

The patient's vWF concentration and vWF-cp activity was compared with controls after the patient's PLT had been restored at day 32 after admission. Results indicated that the vWF concentration was broadly comparable with the controls, but were marginally higher in the patient, which was a likely cause of the elevated vWF-cp activity in the patient in comparison with the healthy volunteers, which is consistent with previous findings (24). However, vWF-cp concentration was lower than expected given the findings of previous studies. Further studies in larger cohorts are required to fully illuminate vWF-cp activity in TTP. According to the current findings, PE is the preferred treatment for TTP and it can markedly decrease the mortality rate of TTP. A standard or low dose of rituximab is effective for treatment of TTP and lowers the recurrence rate. The occurrence of TTP is closely related to $\mathrm{vWF}$ and $\mathrm{vWF}-\mathrm{cp}$.

\section{Acknowledgements}

The present study was supported by the Finance Department of Jilin Province in China (grant no. 3D514L533430).

\section{References}

1. Amorosi EL and Ultmann JE: Thrombotic thrombocytopenic purpura: Report of 16 cases and review of the literature. Medicine 45: 139-159, 1966.

2. Allford SL, Hunt BJ, Rose P and Machin SJ; Haemostasis and Thrombosis Task Force, British Committee for Standards in Haematology: Guidelines on the diagnosis and management of the thrombotic microangiopathic haemolytic anaemias. $\mathrm{Br}$ J Haematol 120: 556-573, 2003.

3. Said A, Haddad RY, Stein R and Lerma EV: Thrombotic thrombocytopenic purpura. Dis Mon 60: 500-504, 2014.

4. Edel E, Al-Ali HK, Seeger S, Kauschat D and Matthes G: efficacy and safety profile of solvent/detergent plasma in the treatment of acute thrombotic thrombocytopenic purpura: A single-center experience. Transfus Med Hemother 37: 13-19, 2010.

5. Ye YF, Wang YS and Shen ZY: National Guide to Clinical Laboratory Procedures. Third Edition. Southeast University Press, Nanjing, 2006.

6. Rovner BW and Folstein MF: Mini-mental state exam in clinical practice. Hosp Pract (Off Ed) 22: 99, 103, 106, 110, 1987

7. Chalmers J: The 1999 WHO-ISH guidelines for the management of hypertension. Med J Aust 171: 458-459, 1999.

8. Bell WR, Braine HG, Ness PM and Kickler TS: Improved survival in thrombotic thrombocytopenic purpura-hemolytic uremic syndrome. Clinical experience in 108 patients. N Engl J Med 325: 398-403, 1991.

9. George JN: How I treat patients with thrombotic thrombocytopenic purpura: 2010. Blood 116: 4060-4069, 2010.

10. Bukowski RM, Hewlett JS, Harris JW, Hoffman GC, Battle JD Jr, Silverblatt E and Yang IY: Exchange transfusions in the treatment of thrombotic thrombocytopenic purpura. Semin Hematol 13: 219-232, 1976
11. Fontana S, Kremer Hovinga JA, Lämmle B and Mansouri Taleghani B: Treatment of thrombotic thrombocytopenic purpura. Vox Sang 90: 245-254, 2006.

12. Rizzo C, Rizzo S, Scirè E, Di Bona D, Ingrassia C, Franco G, Bono R, Quintini G and Caruso C: Thrombotic thrombocytopenic purpura: A review of the literature in the light of our experience with plasma exchange. Blood Transfus 10: 521-532, 2012.

13. Westwood JP, Webster H, McGuckin S, McDonald V, Machin SJ and Scully M: Rituximab for thrombotic thrombocytopenic purpura: Benefit of early administration during acute episodes and use of prophylaxis to prevent relapse. J Thromb Haemost 11: 481-490, 2013

14. Heidel F, Lipka DB, von Auer C, Huber C, Scharrer I and Hess G: Addition of rituximab to standard therapy improves response rate and progression-free survival in relapsed or refractory thrombotic thrombocytopenic purpura and autoimmune haemolytic anaemia. Thromb Haemost 97: 228-233, 2007.

15. Foley SR, Webert K, Arnold DM, Rock GA, Clark WF, Barth D and Sutton DM; Members of the Canada Apheresis Group (CAG): A Canadian phase II study evaluating the efficacy of rituximab in the management of patients with relapsed/refractory thrombotic thrombocytopenic purpura. Kidney Int Suppl: S55-S58, 2009.

16. Tun NM and Villani GM: Efficacy of rituximab in acute refractory or chronic relapsing non-familial idiopathic thrombotic thrombocytopenic purpura: A systematic review with pooled data analysis. J Thromb Thrombolysis 34: 347-359, 2012.

17. Moake JL, Rudy CK, Troll JH, Weinstein MJ, Colannino NM, Azocar J, Seder RH, Hong SL and Deykin D: Unusually large plasma factor VIII: Von willebrand factor multimers in chronic relapsing thrombotic thrombocytopenic purpura. N Engl J Med 307: 1432-1435, 1982.

18. Furlan M, Robles R and Lämmle B: Partial purification and characterization of a protease from human plasma cleaving von willebrand factor to fragments produced by in vivo proteolysis. Blood 87: 4223-4234, 1996.

19. Gerritsen HE, Robles R, Lämmle B and Furlan M: Partial amino acid sequence of purified von willebrand factor-cleaving protease. Blood 98: 1654-1661, 2001

20. Kremer Hovinga JA and Lämmle B: Role of ADAMTS13 in the pathogenesis, diagnosis, and treatment of thrombotic thrombocytopenic purpura. Hematology Am Soc Hematol Educ Program 2012: 610-616, 2012.

21. Duraković N, Radonić R and Gasparović V: Thrombotic thrombocytopenic purpura-the role of ADAMTS13 assay in clinical practice. Coll Antropol 34: 1087-1091, 2010.

22. Kremer Hovinga JA, Vesely SK, Terrell DR, Lämmle B and George JN: Survival and relapse in patients with thrombotic thrombocytopenic purpura. Blood 115: 1500-1511, 2010.

23. Peyvandi F, Lavoretano S, Palla R, Feys HB, Vanhoorelbeke K, Battaglioli T, Valsecchi C, Canciani MT, Fabris F, Zver S, et al: ADAMTS13 and anti-ADAMTS13 antibodies as markers for recurrence of acquired thrombotic thrombocytopenic purpura during remission. Haematologica 93: 232-239, 2008

24. Arya M, Anvari B, Romo GM, Cruz MA, Dong JF, McIntire LV, Moake JL and López JA: Ultralarge multimers of von willebrand factor form spontaneous high-strength bonds with the platelet glycoprotein Ib-IX complex: Studies using optical tweezers. Blood 99: 3971-3977, 2002

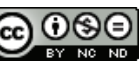

This work is licensed under a Creative Commons Attribution-NonCommercial-NoDerivatives 4.0 International (CC BY-NC-ND 4.0) License. 